\title{
Rechnergestützte, weniger invasive Verfahren bei Osteosynthesen am Becken*
}

\author{
Paul Alfred Grützner, Jochen Franke, Bernd Vock, Andreas Wentzensen
}

\section{Zusammenfassung}

Durch die dreidimensionale Bildgebung mit einem speziellen Bildverstärker lässt sich im Bereich der anatomisch komplexen Region des Beckens eine verbesserte intraoperative Kontrolle des Repositionsergebnisses und der Qualität der Osteosynthese erzielen. Dies ist eine wesentliche Voraussetzung, auch bei reduzierter Invasivität ohne direkte Darstellung der Fraktur die Qualität der Versorgung zu gewährleisten. In Kombination mit der registrierungsfreien Navigation können die Abläufe optimiert werden und versprechen einen weiteren Gewinn an Sicherheit und Qualität bei der Versorgung von Verletzungen am Becken.

\section{Einleitung}

Aufgrund der nicht unerheblichen $\mathrm{Zu}$ gangsmorbidität bei der Versorgung komplexer Beckenverletzungen ist eine Reduktion der Invasivität anzustreben. Die Qualität der Frakturreposition und der Osteosynthese darf hierdurch jedoch nicht beeinträchtigt werden. Die technischen Voraussetzungen sind eine optimierte intraoperative Bildgebung und möglichst die Anbindung an die Navigation. Durch eine Reduktion der Invasivität können jedoch biomechanische Prinzipien nicht außer Kraft gesetzt werden.

Die Reduktion der Invasivität darf nicht zu einem Qualitätsverlust bei der Osteosynthese führen.
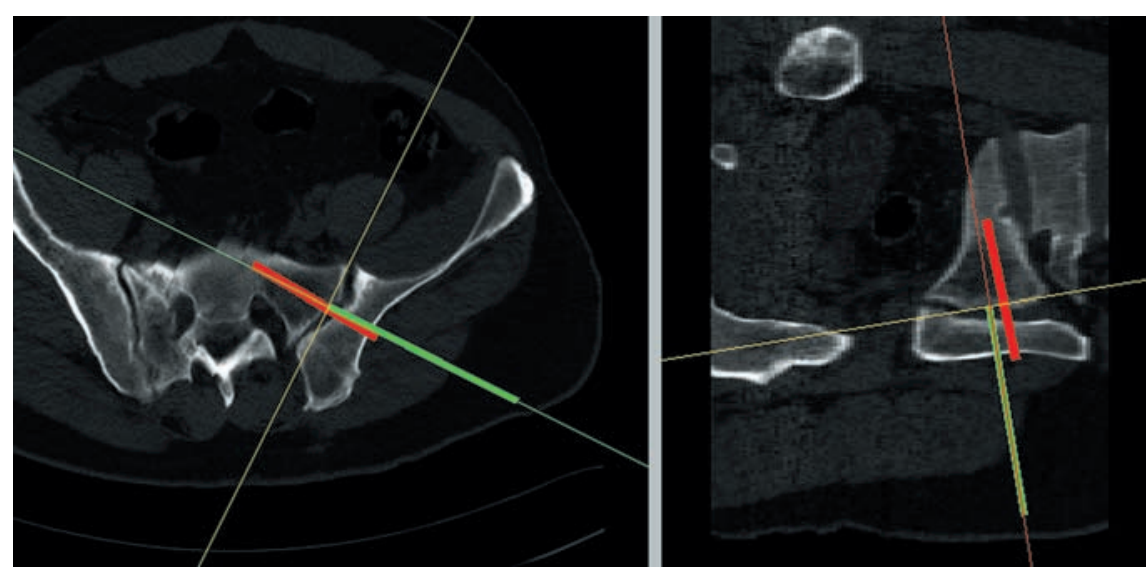

Abb.1 CT-basierte Navigation bei ileosakraler Dübelarthrodese.
Bei Osteosynthesen am Becken stößt die intraoperative zweidimensionale Bildgebung mit einem C-Bogen in vielen Fällen an ihre Grenzen, da komplexe dreidimensionale Knochenstrukturen sich nur unzureichend abbilden lassen. In diesen Fällen bleibt eine Unsicherheit bezüglich der korrekten Rekonstruktion der knöchernen Strukturen und der korrekten Position des Osteosynthesematerials. Gerade bei minimalinvasiven Verfahren, wie zum Beispiel bei der perkutanen Verschraubung er Ileosakralfuge oder perkutanen Verschraubungen am Azetabulum oder am vorderen Beckenring, ist die Orientierung anspruchsvoll und die Rate an fehlplatzierten Schrauben nicht unerheblich $[1,19,20,22,24]$.

\section{CT-basierte Navigation}

Durch die CT-basierte chirurgische Navigation konnte eine Verknüpfung der präoperativ gewonnenen dreidimensionalen Bilddaten mit der intraoperativen Instrumentenposition erreicht werden. Damit die Koordinaten der Instrumente

* Herrn Prof. Dr. med. Dr. h.c. mult. Siegfried in den Datensatz und damit auf die Anatomie des Patienten übertragen werden können, ist ein Registrierungsprozess erforderlich, der in den meisten Fällen manuell durchgeführt wird, indem mit einem navigierten Instrument am Patienten Landmarken aufgesucht werden, die im präoperativen CT geplant wurden.

Die wesentliche Limitierung in der CT basierten Navigation besteht in diesem Registrierungsprozess. Das Auffinden definierter Landmarken am Becken ist aufwendig, schwierig und daher häufig ungenau und mit zusätzlicher Invasivität verbunden. Verbessert werden kann dieser Prozess durch präoperativ, vor der Aufnahme des CT, eingebrachte Marker (fiducials), die sich intraoperativ mit ge-

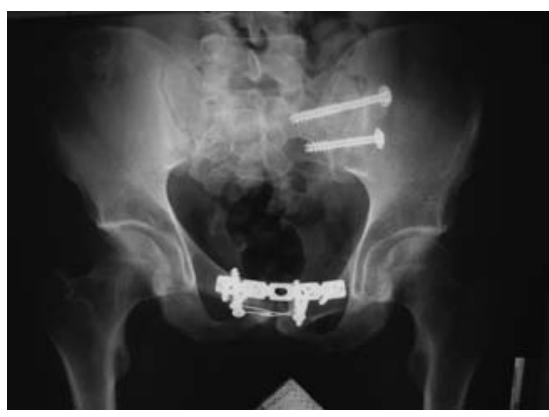

Abb. 2 Postoperatives Bild der IS-Arthrodese.
OP-JOURNAL 2003; 19: 168-173

(C) Georg Thieme Verlag Stuttgart . New York 
ringer Mühe auffinden lassen. Dieses Marker matching ist allerdings mit einem zusätzlichen Eingriff verbunden und somit speziellen Indikationen, wie zum Beispiel bei aufwändigen Korrektureingriffen nach fehlverheilten Frakturen oder nach Nofallstabilisierung mittels Fixateur externe vorbehalten.

Da auch Änderungen der Anatomie aber intraoperativ nicht erfasst werden können, sind die Anwendungsgebiete der CT-basierten Navigation beschränkt $[4,9,11,17]$. Nur in wenigen Einrichtungen ist es möglich, mit einer direkten CT-Anbindung navigierte Eingriffe am Becken durchzuführen. Nach Änderung der Anatomie können die CT-Daten jeweils aktualisiert werden (Modalitätsbasierte Navigation) [3,16]. Erst durch spezielle Registrierungsverfahren und Segmentierung des CT-Datensatzes in die einzelnen Frakturfragmente ist es möglich, die Frakturreposition am Becken virtuell zu verfolgen. Die ersten experimentellen Ansätze sind viel versprechend [12].

Die CT-basierte Navigation liefert die beste Bildqualität, ist aber durch den Registrierungsprozess und die Einschränkungen bei Veränderungen in der Anatomie nur bedingt einsetzbar.

\section{Virtuelle Fluoroskopie}

Bei der Navigation in 2D-C-Bogen-Daten, der sog. virtuellen Fluoroskopie, wird nicht nur das chirurgische Instrument, sondern auch der C-Bogen mit Markern bestückt und von der Kamera des Navigationssystems verfolgt. Es können bis zu vier Bildwandlerebenen gleichzeitig in einer Art virtueller Dauerdurchleuchtung als optische Information im Operationssaal zur Verfügung stehen. Die Registrierung läuft intraoperativ automatisch $a b$, es ist daher kein Matching erforderlich. Bei Änderungen der Anatomie können daher unkompliziert neue Aufnahmen unabhängig von der Position des C-Bogens oder der Patientenlage durchgeführt und in das Navigationssystem transferiert werden.

Die Bildgebung ist auf die zweidimensionalen Informationen aus dem C-Bogen beschränkt. Limitierungen bestehen daher bei komplexen anatomischen Strukturen des Beckens $[10,11,18]$. So ist beispielsweise die Darstellung der Foramina sacraliae und des Spinalkanals häufig nicht sicher möglich. Bezüglich der Bildqualität müssen bei der C-Bogen-basierten 2D-Navigation gerade bei adipösen

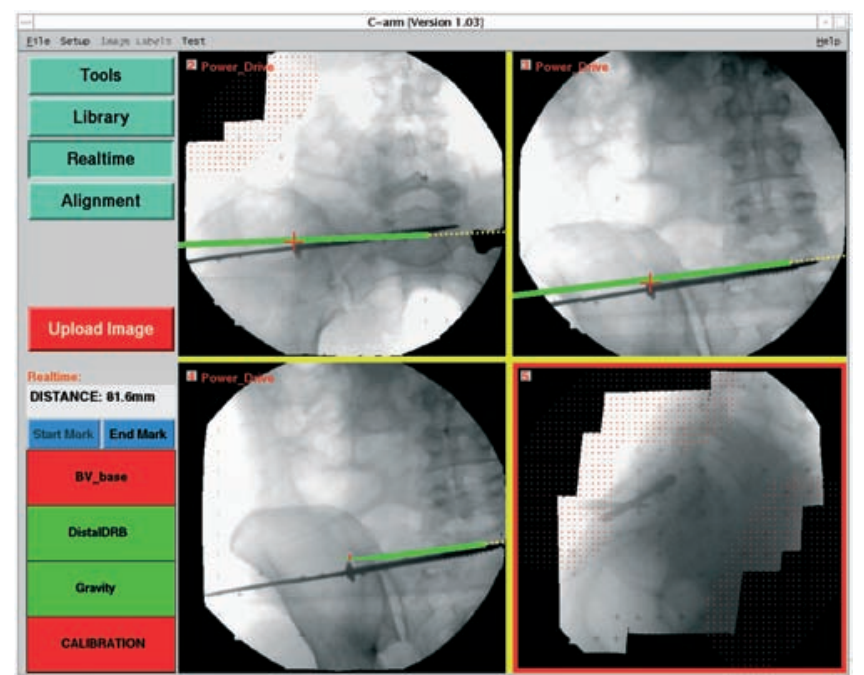

Abb. 3 2D-Navigation mit synchroner Darstellung in 4 Ebenen.

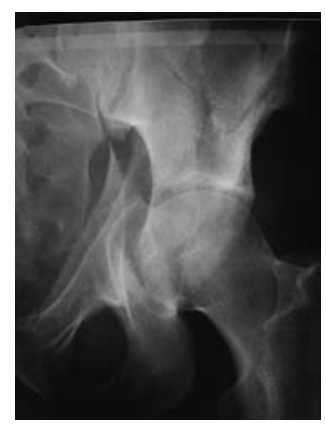

Abb. 4 Azetabulumfraktur.

und geblähten Patienten Abstriche gemacht werden. Außerdem kann die korrekte Einstellung des C-Bogens zur Herausforderung für den Operateur und das Pflegepersonal werden.

Die 2D-Navigation ist einfach anzuwenden, und intraoperativ können bei veränderter Anatomie jederzeit neue Aufnahmen in das Navigationssystem geladen werden. Der Nachteil liegt am Becken in dem eingeschränkten Informationsgehalt von zweidimensionalen Bildern und der Bildqualität.

\section{D-Bildverstärker-Technologie}

Mit dem mobilen isozentrischen 3D-CBogen SIREMOBIL ${ }^{\circledR}$ Iso-C ${ }^{3 D}$ (Siemens AG, Medical Solutions, Erlangen) steht erstmals ein Gerät zur Verfügung, das ohne Mehraufwand die intraoperative dreidimensionale Darstellung knöcherner Strukturen erlaubt. Hierdurch wird eine unmittelbare Prozess- und Ergebniskontrolle des rekonstruktiven Eingriffs möglich, so dass bereits intraoperativ Konsequenzen aus der Bildgebung gezogen werden können [5-8,13,21].

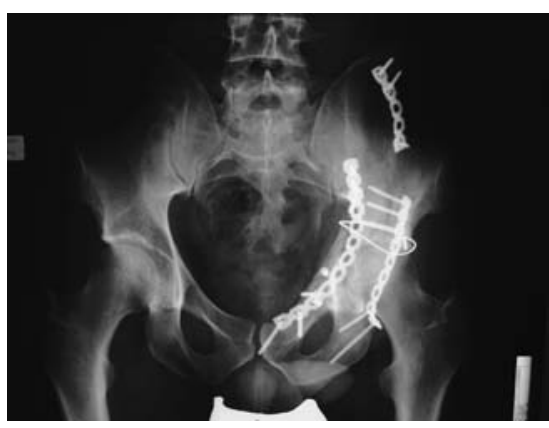

Abb. 5 Nach Versorgung über kombinierten Zugang.

Die intraoperative 3D-Bildgebung von SIREMOBIL Iso- $\mathrm{C}^{3 \mathrm{D}}$ basiert auf einer isozentrischen $190^{\circ}$-Orbitalbewegung des Bildverstärkers. Neben der konventionellen 2D-Bildgebung kann im 3D-Betrieb während einer motorgesteuerten kontinuierlichen orbitalen Rotation um $190^{\circ}$ eine definierte Zahl von Durchleuchtungsbildern in festen Winkelabständen aufgenommen werden. Simultan errechnet die 3D-Workstation im Monitorwagen des C-Bogens einen hochaufgelösten, isotropen 3D-Datenwürfel im Isozentrum mit einer Kantenlänge von etwa $12 \mathrm{~cm}$. Unmittelbar nach der Datenaufnahme kann der Chirurg beliebige MPR-Schnittebenen durch diese Daten in Echtzeit legen. Zusätzlich stehen die zweidimensionalen sequenziellen Röntgenprojektionen als Filmsequenz zur Verfügung.

Die intraoperative 3D-Bildgebung liefert wichtige Informationen in anatomisch komplexen Regionen. Aufgrund der ausschließlich möglichen Hochkontrastdarstellung können im Gegensatz zum CT nur knöcherne Strukturen dargestellt werden. 


\section{Registrierungsfreie Navigation mit 3D Bilddaten}

Durch die Anbindung des SIREMOBIL Iso$\mathrm{C}^{3 \mathrm{D}}$ an die Navigation ist es möglich, die erzeugten 3D-Daten im DICOM-Format, inklusive der Raumkoordinaten, direkt an das angekoppelte Navigationssystem zu übertragen. Die Vorteile der CT-basierten Navigation mit der dreidimensionalen Darstellung der knöchernen Strukturen werden also mit den Vorteilen einer registrierungsfreien Navigation mit intraoperativer Bildgebung verknüpft. Das chirurgische Instrument wird sofort, d.h. ohne eventuelle aufwendige manuelle Registrierungsprozedur, im Bild angezeigt. Fehlerquellen durch eine mögliche schlechte Registrierung werden ver-

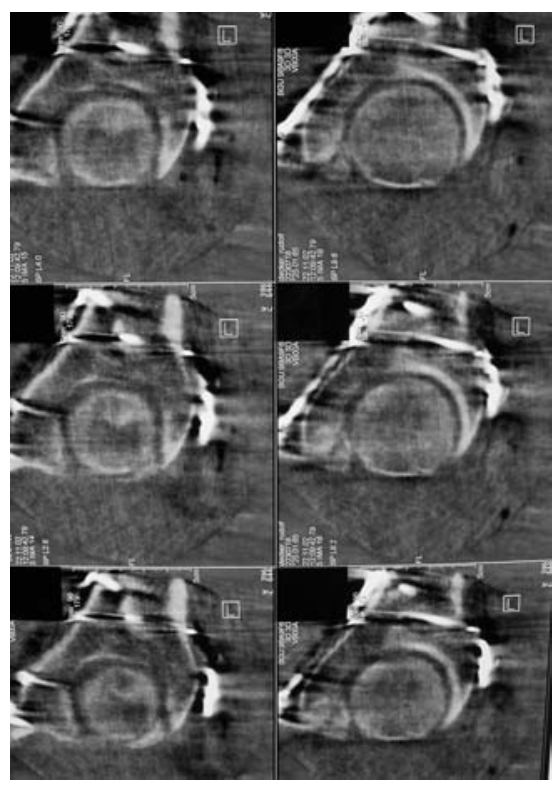

Abb. 6 Intraoperative 3D-Bildgebung, multiplanare Rekonstruktionen des Azetabulums. mieden und neue Röntgenbilder können intraoperativ erzeugt werden.

Seit Januar 2002 besteht in der Berufsgenossenschaftlichen Unfallklinik Ludwigshafen die Möglichkeit der registrierungsfreien 3D-Navigation durch die Anbindung des SIREMOBIL Iso-C ${ }^{3 D}$ an das SurgiGATE-Navigationssystem.

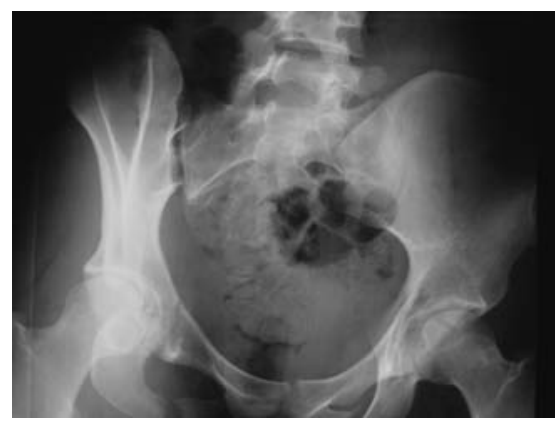

Abb. 8 C1 Beckenfraktur mit kompletter Ruptur der IS Fuge rechts.
Die Patienten werden auf einem metallfreien Karbontisch gelagert. Aufgrund der registrierungsfreien Navigation, ohne die Notwendigkeit der manuellen Landmarkendefinition, sind die Voraussetzungen für ein minimal invasives Vorgehen gegeben.

Die Navigation erfolgte dann nach einem standardisierten Schema. Vor dem 3D-

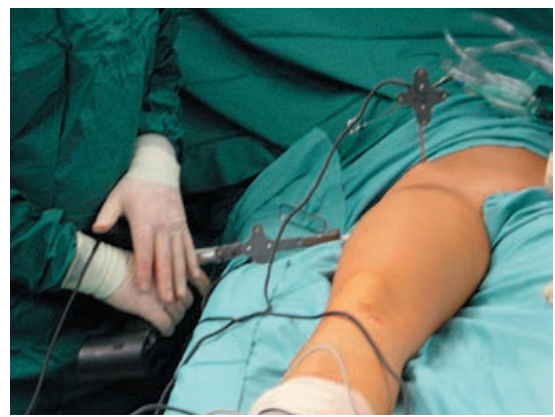

Abb.9 Perkutane Verschraubung der ISFuge.

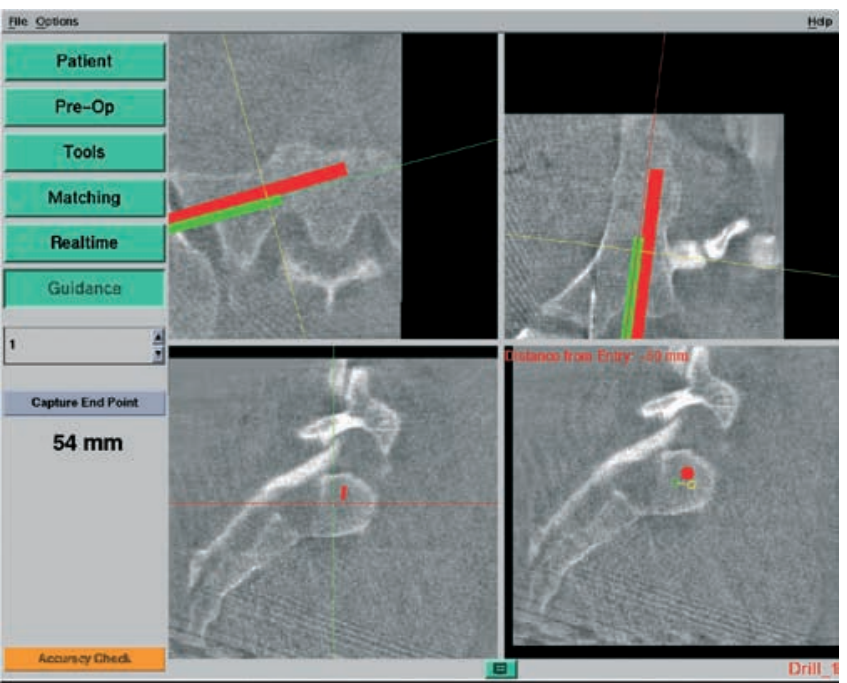

Abb.10 Geplante (rot) und aktuelle (grün) Position des Bohrers.
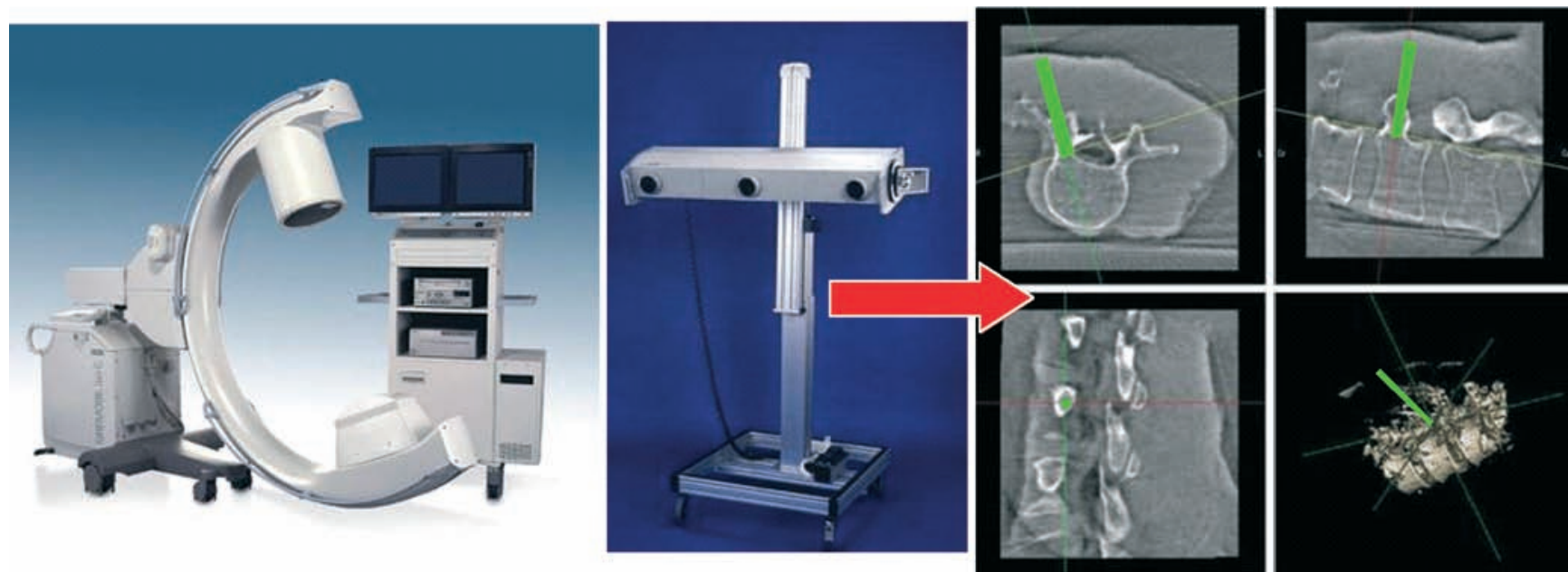

Abb. 7 Prinzip der Navigation in 3D-Bildwandlerdaten. 


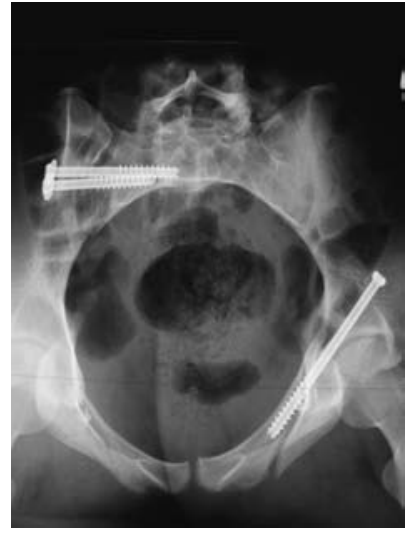

Abb.11 Postoperative Kontrolle, der vordere Pfeiler links wurde retrograd navigiert verschraubt.

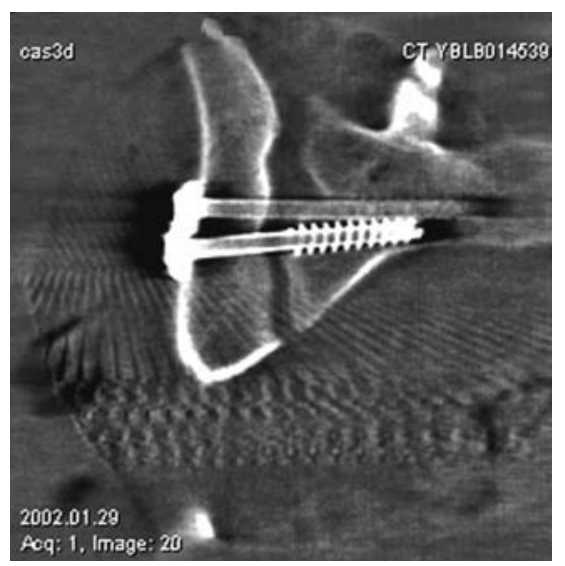

Abb.12 Intraoperative Kontrolle der IS-Verschraubung (multiplanare Rekonstruktion).

Scan wird die DRB am Becken fixiert. Dann werden die ISO-C ${ }^{3 D}$-Bilder aufgenommen. Hierbei muss gewährleistet sein, dass die Startposition des Bildverstärkers und die DRB am Patienten für die Kamera des Navigationssystems gleichzeitig sichtbar sind. Nach dem Transfer der Daten in das Navigationssystem wird die Präzision des Systems über einen so genannten „Verification“-Modus durch den Operateur kontrolliert. In diesem Modus überprüft der Operateur die Instrumentenposition, indem er das Instrument an eine klar zu identifizierende anatomische Struktur hält und die Übereinstimmung zwischen anatomischer Struktur und der angezeigten Position des Instrumentes im Navigationssystem auf Plausibilität überprüft.

Auch bei Azetabulumfrakturen kann unter der Voraussetzung der Möglichkeit zur geschlossenen Reposition der Fraktur, minimal invasiv navigiert die Osteosyn-

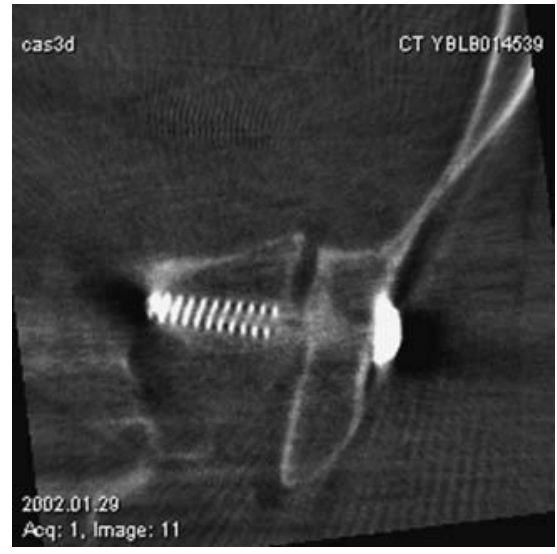

Abb.13 Intraoperative Kontrolle der IS-Verschraubung (multiplanare Rekonstruktion).

these erfolgen. Zur Anwendung kommen hier alle Techniken aus dem Repertoire der minimal invasiven Reposition mit Einsatz von Traktion, Schanzschrauben als Joystick und Zugschrauben. Der wesentliche Vorteil des intraoperativen Einsatzes der 3D-Bildgebung ist die Möglichkeit der direkten Kontrolle des Repositionsergebnisses. Dennoch sind minimal invasive Verfahren am Azetabulum derzeit noch wenigen Indikationen vorbehalten. Der zunehmende Einsatz dieser Technik führt aber auch bei den konventionellen Zugängen zu einer Reduktion der Invasivität.

Durch intraoperative 3D-Bildgebung in Kombination mit der registrierungsfreien Navigation können Sicherheit und Qualität bei der Versorgung von Verletzungen am Becken optimiert werden.

\section{Klinische Ergebnisse}

Bei 48 Patienten kam intraoperativ der ISO-C ${ }^{3 \mathrm{D}}$ bei Versorgungen von Beckenverletzungen zum Einsatz. Bei 39 Patienten erfolgte bei offenen Osteosynthesen, vornehmlich am Azetabulum der diagnostische Einsatz zur Kontrolle der Frakturreposition und der Lage des Osteosynthesematerials. Bei 5 dieser Patienten hatten die Informationen aus dem ISO-C ${ }^{3 \mathrm{D}}$ unmittelbare intraoperative Konsequenzen. In einem Fall wurde die Frakturreposition korrigiert, in einem Fall der zunächst nur ventrale Zugang aufgrund einer verbliebenen Dislokation am dorsalen Pfeiler ausgedehnt und in 3 Fällen eine intraartikulär liegende Schraube getauscht oder ersatzlos entfernt.

Bei bislang 9 Patienten erfolgten navigierte Schraubenplatzierungen am Be-

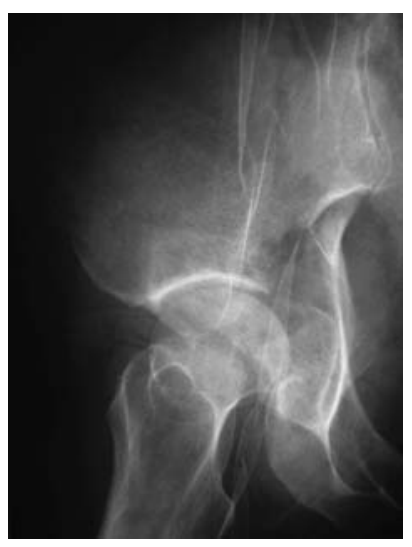

Abb.14 Aztetabulumfraktur A3 (vorderer Pfeiler).

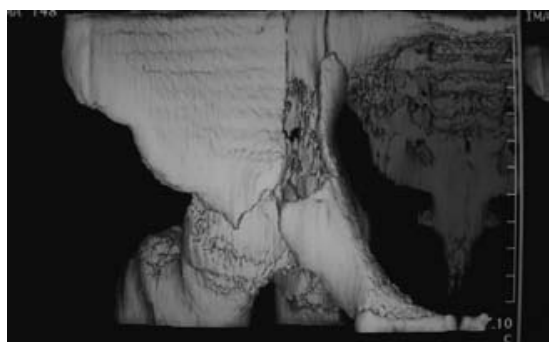

Abb.15 Präoperative CT-Rekonstruktion.

ckenring und Azetabulum (IS-Fugenverschraubung, Verschraubung des vorderen Pfeilers, Azetabulumosteosynthese). Insgesamt wurden 28 Schrauben navigiert eingebracht. In drei Fällen wurden zwei 3D-Datensätze zur Navigation erzeugt. Weitere Scans wurden zur unmittelbar postoperativen Kontrolle der Implantatposition im OP aufgenommen. In der Beckengruppe lagen alle Schrauben in den postoperativen Kontrollen korrekt. Die mittlere Durchleuchtungszeit für die Aufnahme der 3D-Scans und die 2D-Kontrolle liegt bei $1,38 \mathrm{~min}$.

\section{Diskussion}

In dieser Studie konnte erstmals die klinische Anwendung der Navigation in dreidimensionalen Datensätzen aus dem SIREMOBIL Iso- $\mathrm{C}^{3 \mathrm{D}}$ mit automatischer Registrierung am Becken gezeigt werden.

In der Literatur wird bei konventionellen Techniken eine relativ hohe Rate an Fehlplatzierungen von bis zu $40 \%$ beschrieben. Gerade am Becken kann eine Fehlplatzierung von Implantaten zu nicht unerheblichen langfristigen Komplikationen bezüglich der Stabilität und der Verletzung von Gefäß- und Nervenstruktu- 

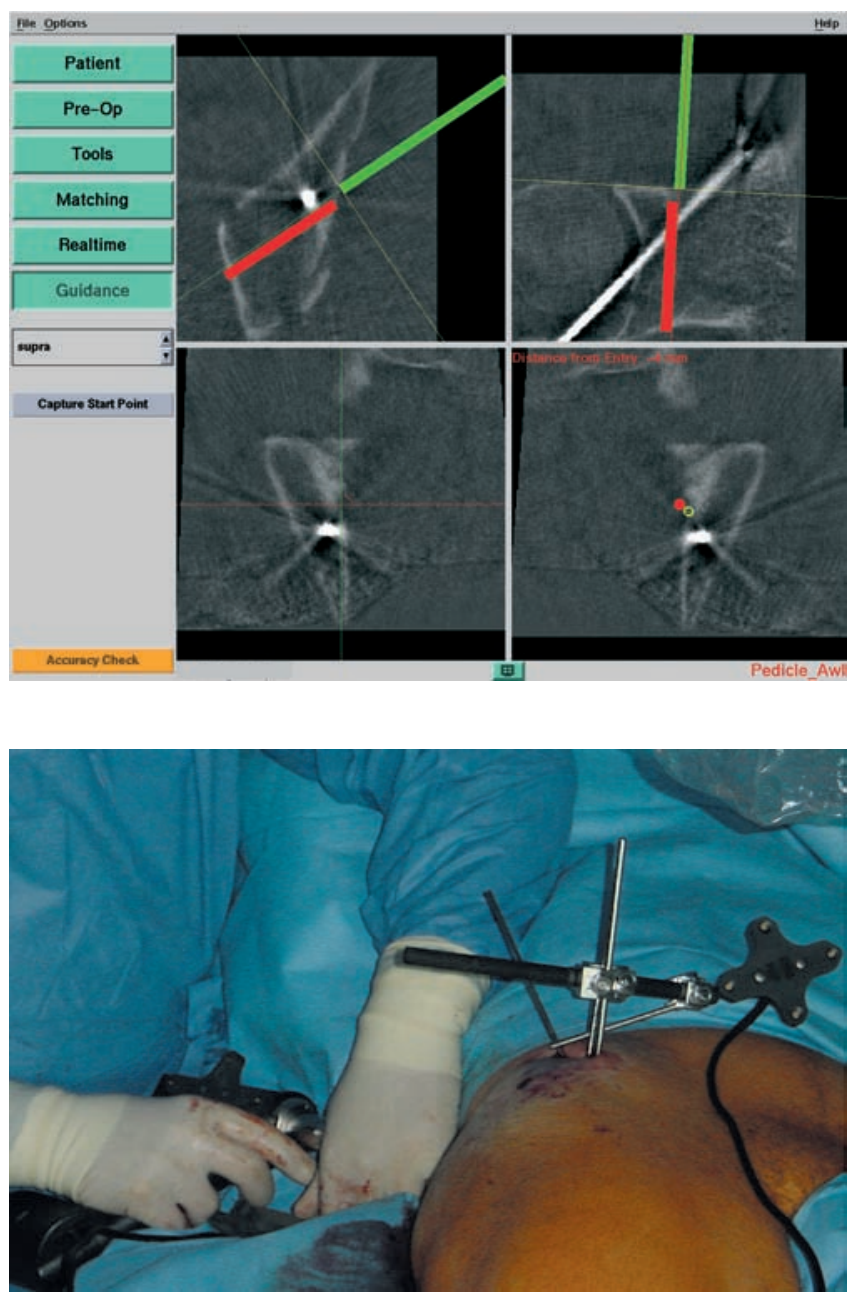

Abb.17 Perkutane Verschraubung, die eingebrachten

Schanzschrauben wurden als Repositionshilfe und zur Befestigung der DRB genutzt.

Abb.16 Intraoperative Navigation.

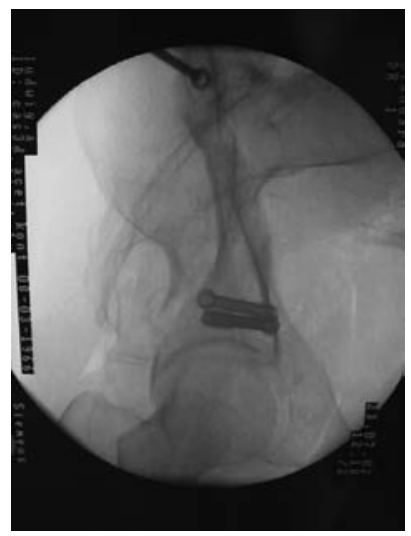

Abb.18 Intraoperative 2D-BV-Kontrolle.

ren führen. Die konventionelle Technik verlangt daher vom Operateur eine große Erfahrung, da die Positionierung der Schraube ohne direkte visuelle Kontrolle erfolgen muss. Das Ziel eines Navigationssystems ist es, die Präzision der Schraubenpositionierung $\mathrm{zu}$ verbessern und die intraoperativen Fehlbohrungen

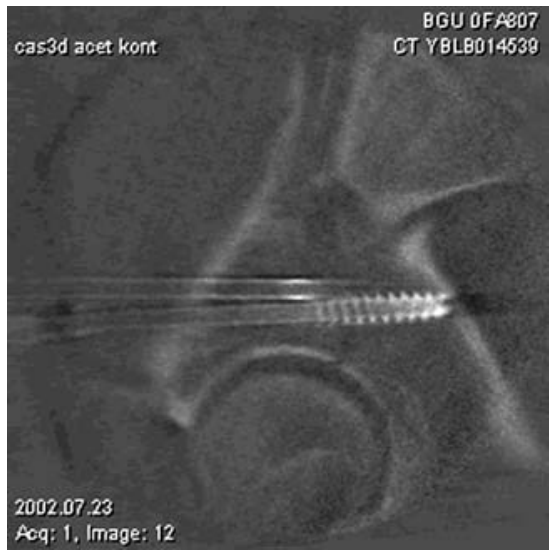

Abb.20 Intraoperative multiplanare Rekonstruktion (MPR) mit nahezu stufenfreier Reposition.

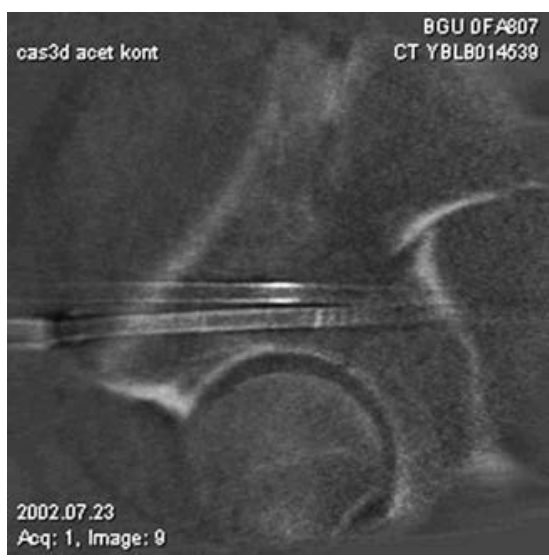

Abb. 21 MPR.

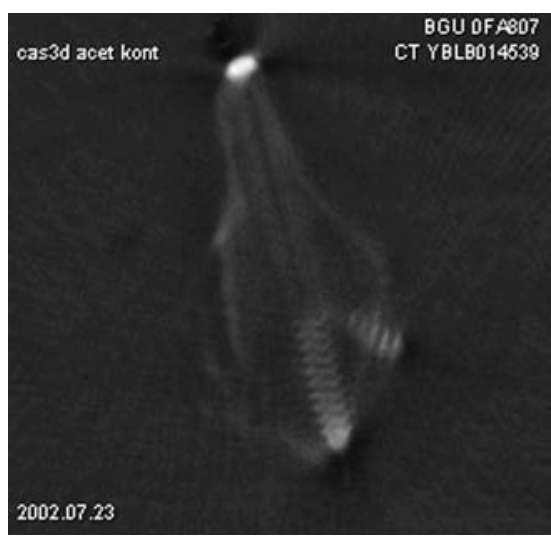

Abb. 22 Supraazetabuläre MPR.

und Korrekturen zu reduzieren. Nicht zuletzt soll die, bei Osteosynthesen am Becken in der Regel hohe intraoperative Strahlenbelastung für den Patienten, aber auch für das medizinische Personal, reduziert werden.

Durch CT-basierte und C-Bogen-basierte Navigationssysteme konnte die Qualität von Schraubenplatzierungen in anatomisch komplexen Regionen am Bewegungsapparat verbessert und die intraoperative Durchleuchtungszeit reduziert werden $[2,4,15,23]$. Beide Verfahren unterliegen jedoch systemimmanenten Li- 
mitierungen. Bei der Iso- $\mathrm{C}^{3 \mathrm{D}}$-Navigation unterliegen die Durchleuchtungszeiten aufgrund des sehr standardisierten Vorgehens sehr geringen Schwankungen. Besonders zu beachten ist, dass bei der Aufnahme des 3D-Datensatzes sich das gesamte OP-Personal außerhalb des Kontrollbereiches aufhalten kann, während sich bei der konventionellen Vorgehensweise der Operateur meist unmittelbar neben der Strahlenquelle aufhält.

Der wichtigste Aspekt ist sicherlich die Präzision der navigiert durchgeführten Aktionen. Dies hat dazu geführt, dass die Iso-C ${ }^{3 D}$-Navigation am Becken die anderen Verfahren verdrängt hat. Der Einsatz ist aber immer noch durch den hohen Schulungsaufwand eingeschränkt. Die computerunterstützen Verfahren führen zu einem Zugewinn an intraoperativer Präzision, wobei - wie hier gezeigt - die größte Genauigkeit mit der neuen Iso- $\mathrm{C}^{3 \mathrm{D}}$-Navigation erreicht wird.

\section{Literatur}

${ }^{1}$ Altman DT, Jones CB, Routt ML Jr. Superior gluteal artery injury during iliosacral screw placement. J Orthop Trauma 1999; 13: 220 7

2 Arand M, Hartwig E, Hebold D, Kinzl L, Gebhard F. [Precision analysis of navigation-assisted implanted thoracic and lumbar pedicled screws. A prospective clinical study]. Unfallchirurg 2001; 104: 1076-81

${ }^{3}$ Berlemann U, Langlotz F, Langlotz U, Nolte LP. [Computer-assisted orthopedic surgery. From pedicle screw insertion to further applications]. Orthopade 1997; 26: $463-9$

${ }^{4}$ Berlemann U, Monin D, Arm E, Nolte LP, Ozdoba C. Planning and insertion of pedicle screws with computer assistance. J Spinal Disord 1997; 10: 117-24

${ }^{5}$ El Sheik M, Heverhagen JT, Alfke H et al. Multiplanar Reconstructions and Three-dimensional Imaging (Computed Rotational Osteography) of Complex Fractures by Using a Carm System: Initial Results. Radiology 2001; 221: $843-9$

${ }^{6}$ Euler E, Wirth S, Linsenmaier U, Mutschler W, Pfeifer KJ, Hebecker A. Vergleichende Untersuchung zur Qualität C-Bogen-basierter 3D-Bildgebung am Talus. Unfallchirurg 2001; 104: 839-46

${ }^{7}$ Euler E, Heinig S, Fischer T, Pfeifer KJ, Mutschler W. Erste klinische Erfahrungen mit dem SIREMOBIL Iso-C ${ }^{3 D}$. electromedica 2002; 70: 64-7

${ }^{8}$ Euler E, Wirth S, Pfeifer KJ, Mutschler W, Hebecker A. 3D-Bildgebung mit einem mobilen isozentrischen C-Bogen. electromedica 2000; 68: $122-6$

${ }^{9}$ Gebhard F, Kinzl L, Arand M. Grenzen der CTbasierten Computernavigation in der Wirbelsäulenchirurgie. Unfallchirurg 2000; 103: $696-701$
${ }^{10}$ Grützner PA, Köhler T, Vock B, Wentzensen A. Rechnergestütztes Operieren an der Wirbelsäule. OP Journal 2001; 17: 185-90

${ }^{11}$ Grützner PA, Vock B, Wentzensen A. Use of computer assisted, fluoroscopy based navigation in treatment of pelvic ring disruptions. In: Lemke HU, Vannier MW, Inamura K, Farman AG, Doi K, eds. Computer Assisted Radiology and Surgery. Elsevier Science B.V., 2000: 965

${ }^{12}$ Hufner T, Pohlemann T, Tarte S et al. Computer-assisted fracture reduction of pelvic ring fractures: an in vitro study. Clin Orthop 2002; $231-9$

${ }^{13}$ Kotsianos D, Rock C, Wirth S et al. [Detection of tibial condylar fractures using 3D imaging with a mobile image amplifier (Siemens ISO-C-3D): Comparison with plain films and spiral CT]. Rofo Fortschr Geb Rontgenstr Neuen Bildgeb Verfahr 2002; 174: 82 -7

${ }^{14}$ Laine T, Lund T, Ylikosi M, Lohikoski J, Schlenzka D. Accuracy of pedicle screw insertion with and without computer assistance: a randomised controlled clinical study in 100 consecutive patients. E Spine J 2000; 9: 235-40

${ }^{15}$ Laine T, Schlenzka D, Mätikalo K, Tallroth K, Nolte L-P, Visarius H. Improved Accuracy of Pedicle Screw Insertion With Computer-Assisted Surgery. Spine 1997; 11: 1254-8

${ }^{16}$ Messmer P, Baumann B, Suhm N, and Jacob AL. [Navigation systems for image-guided therapy: A review]. Rofo Fortschr Geb Rontgenstr Neuen Bildgeb Verfahr 2001; 173: $777-84$

${ }^{17}$ Nolte LP, Zamorano LJ, Jiang Z, Wang Q Langlotz F, Berlemann U. Image-guided insertion of transpedicular screws. A laboratory set-up. Spine 1995; 20: 497-500

${ }^{18}$ Nolte LP, Zamorano LJ, Jiang Z, Wang $\mathrm{Q}$ Langlotz F, Berlemann U. Image-guided insertion of transpedicular screws. A laboratory set-up. Spine 1995; 20: 497-500

${ }^{19}$ Reilly MC, Bono CM, Litkouhi B, Sirkin M, Behrens FF. The effect of sacral fracture malreduction on the safe placement of iliosacral screws. J Orthop.Trauma 2003; 17: 88-94

${ }^{20}$ Ricci WM, Padberg AM, Borrelli J. The significance of anode location for stimulus-evoked electromyography during iliosacral screw placement. J Orthop.Trauma 2003; 17: $95-9$

${ }^{21}$ Rock C, Linsenmaier U, Brandl $R$ et al. Vorstellung eines neuen mobilen C-Bogen-/ CT-Kombinationsgerätes (ISO-C-3D): Erste Ergebnisse der 3D-Schnittbildgebung. Der Unfallchirurg 2001; 104: 827-33

${ }^{22}$ Routt ML, Jr., Simonian PT, Mills WJ. Iliosacral screw fixation: early complications of the percutaneous technique. J Orthop Trauma 1997; $11: 584-9$

${ }^{23}$ Suhm N, Jacob AL, Nolte LP, Regazzoni P, Messmer P. Surgical navigation based on fluoroscopy-clinical application for computer-assisted distal locking of intramedullary implants. Comput Aided Surg 2000; 5: 391 400

${ }^{24}$ Tonetti J, Carrat L, Lavallee S, Pittet L, Merloz P, Chirossel JP. Percutaneous iliosacral screw placement using image guided techniques. Clin Orthop 1998; 103-10

\section{Abkürzungen}

$\mathrm{CT}=$ Computertomographie

$\mathrm{DRB}=$ dynamische Referenzierungsbasis

$\mathrm{MPR}=$ multiplanare Rekonstruktionen

MRT $=$ Magnetresonanztomographie

\section{Dr. med. Paul Alfred Grützner}

Oberarzt

Dr. med. Jochen Franke

Assistenzarzt

Dr. med. Bernd Vock

Leitender Oberarzt

Prof. Dr. med. Andreas Wentzensen

Ärztl. Direktor

BG Unfallklinik Ludwigshafen

Klinik für Unfall- und Wieder-

herstellungschirurgie an der

Universität Heidelberg

Ludwig-Guttmann-Str. 13

D-67071 Ludwigshafen 